\title{
An Application of Random Process for Controlled Object Identification with Traffic Delay Problem
}

\author{
$\mathrm{Vu}$ Tien Viet \\ 47 - Dai An - Van Quan, Ha Dong district, Ha Noi city, Vietnam
}

Received: March 17, 2014 / Accepted: April 20, 2014 / Published: February 25, 2015.

\begin{abstract}
In the article proposed an effective method estimating transfer function model of controlled plant including dead-time delay, based on stochatstic time series of input-output signals. The model structure is modified with parameters optimized until the model error becomes "white-noise" series that with inough smal auto-correlation function.
\end{abstract}

\section{Propose}

The Real signals which occur in the control process always imlpy influences of many random factors, so the Directive Object Identification Problem is often related to random process. Mathematically, the Controlled Object Identification problem is the problem that predicts the trend of Random Process: $y(t)=f(t, u)+v(t)$, where $t$ - time; $u$ - vector of non-random input variables; $f(t, u)$ - regressive function that reflects the trend of non-random process or is the model of the identification problem; $u(t)$ random error.

The Theory of Prediction and Identification has been studied and developed with thousands of scientific works made public since last century. We can find the fundamental results of studies of statistics and prediction in $[1,2]$, of kinetics system identification in detail in $[3,4]$.

To use linear algebra methods, we often try to change the regressive models into linear combination forms of coefficients: $f(t, u)=\sum_{i=1}^{n} c_{i} f_{i}(t, u)$, where $c_{i}$-parameters, $f_{i}(t, u)$ - given component functions. By using this model, the Parameter

Corresponding author: Vu Tien Viet, 47 - Dai An - Van Quan, Ha Dong district, Ha Noi city, Vietnam. E-mail: vutietiviet.56@gmail.com.
Identification Problem can be solved easily.

However, this model is not used to solve the analysis and synthesise problem of systems and we have to transform this model into the form of sets of state equations (sets of Cauchy differential equations) or transfer function form. There is a close, easy to exchange relation between set of state equations and transfer function. The transfer function's model of controlled object is often in the following form:

$$
W(C, s)=\frac{b_{0}+b_{1} s+\cdots+b_{m} s^{m}}{a_{0}+a_{1} s+\cdots+a_{n} s^{n}} \cdot e^{-\tau s}, m \leq n
$$

where $s$ - complex number, $\tau \geq 0$ - the dead time delay; $m, n$ - degree of numerators and denominators; $C=\left\{\tau, b_{o}, b_{1}, \cdots, b_{m}, a_{o}, a_{1}, \cdots, a_{n}\right\}$ vector of parameters to be determined.

In the classic works of identification, all the authors concentrated on developing identification methods based on pure polynomial fraction models without the dead time delay components (i.e. set $\tau=0$ ). In fact, exists $\tau \neq 0$, we normally try to use approximate polynomial fraction models with higher degrees of $m, n$ to increase the model accuracy. With this approach, the object identification problem without dead time delay is considered to be completely solved in theory $[1,4]$.

In fact, however, applying the pure polynomial 
fraction methods to the objects with dead time delay is reluctant and ineffective in controlling technology processes such as energy, metallurgy,... because most of the objects obviously have the dead time delay. To have the necessary model accuracy, we normally increase the degree of polynomial fraction to a great value, and therefore making the synthesise problem of systems more complex, even lose its essence.

Disregarding the characteristics of dead time delay of an object is one of the reasons that leads to a great number of research directions of control theory impractically developed, even caused a "crisis" in the previous century [5]. To accurately reflect the controlled object, we have to consider dead time delay as an existing parameter included in the model. Whereas, clearly, the model is non-linear for the parameters. In this case, classic methods are either ineffective or inapplicable.

Because of the above reasons, in order to increase the applicability, we recommend a controlled object identification method based on using directly model (1) along with the dead time delay $\tau$ and other parameters. The following method is based on considering the time response of the object as a random data series.

\section{Estimation of the Object Model from Output Response Data Series}

Suppose the controlled object has weight funtion $w(t)$ with effect input $u(t)$ - predetermined, output response is measured: $y(t)=x(t)+v(t)$, where $v(t)$ - additive noise (figure 1).

Without loss of generality, we restrict $v(t), u(t)$ being the non-intercorrelation scalars, where $v(t)$ is
White Noise, $u(t)$ is step pulse:

$$
u(t)=\left\{\begin{array}{lll}
0 & \text { when } & t \leq 0 \\
1 & \text { when } & t>0
\end{array}\right.
$$

Formerly [3, 4], so as to solve the model estimation problem, we based on popular relation between output response $x(t)$ of object and input signal $u(t)$ :

$$
x(t)=\int_{0}^{t} w(\xi) u(t-\xi) d \xi
$$

where $w(t)$ is the weight function.

From (3), we establish the Problem of Defining weight funtion $w(t)$ upon least square condition

$$
\sigma^{2}=\int_{0}^{T}\left(y(t)-\int_{0}^{t} w(\xi) u(t-\xi) d \xi\right)^{2} \rightarrow \min
$$

and then, define the transfer function $W(s)$ from the weight funtion $w(t)$.

If we parameterize the funtion $w(t)$ in the form of linear combination, the (4) problem will become linear to coefficients and can be solved easily. However, in this way it is complex to select component functions and causes the problem bigger, and therefore makes the problem illconditioned.

To avoid this drawback, we recommend directly using the model of transfer function in form (1) and solve the model estimation problem based on the inverse Laplace transformation. Indeed, if we consider arguments in the Laplace image domain, we obtain:

$$
X(\mathbb{C}, s)=
$$

$$
W(\mathbb{C}, s) \cdot U(s)=\frac{b_{0}+b_{1} s+\cdots+b_{m} s^{m}}{1+a_{1} s+\cdots+a_{n} s^{n}} \cdot \frac{e^{-\tau s}}{s^{q}} \cdot U(s)
$$

where $\quad X(s)=L\{x(t)\}, W(\mathbb{C}, s)=L\{w(t)\}$, $U(s)=L\{u(t)\} ; s$ - complex variable; $L\{\cdot\}$ -

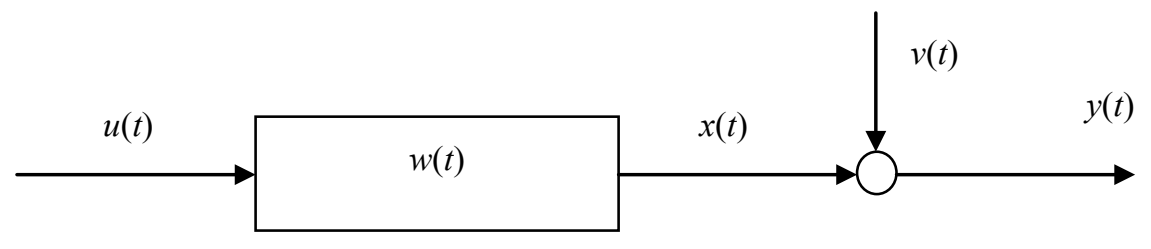

Fig. 1 Linear control system under random effect. 
notation for Laplace transformation, from any $g(t)$ function in real domain $(g(t)=0, \forall t \leq 0)$ into corresponding image $G(s)$ in complex domain.

According to the Inverse Laplace transformation, in [6] we infered a simple formula to compute time response $x(t)$ from its Laplace image:

$$
\begin{aligned}
& x(C, t)=\frac{1}{2 \pi j} \int_{\varrho-j \infty}^{\varrho+j \infty} X(\mathbb{C}, s) e^{s t} d s \\
& =\frac{2 e^{\varrho t}}{\pi} \int_{0}^{\infty} P(\mathbb{C}, \omega) \cos (\omega t) d \omega
\end{aligned}
$$

where $\varrho$ - converging abscissa of Laplace integral (if objects are stable, we can select $\varrho>0$ small enough, for instance $\varrho=0.01 ; \quad j^{2}=-1$, $P(\mathbb{C}, \omega)=\operatorname{Re}\{X(\mathbb{C}, \omega+j \infty)\}$ - the real part of $X(\mathbb{C}, \omega+j \infty)\}$.

Select the upper limit $\left(\omega_{M}\right)$ of the integral which is big enough, then trasform into approximate sum form, we obtain:

$$
x(\mathbb{C}, t)=\frac{2 e^{\varrho t}}{\pi} \sum_{r=1}^{M} P\left(\mathbb{C}, \omega_{r}\right) \cos \left(\omega_{r} t\right)\left[\omega_{r}-\omega_{r-1}\right](7)
$$

where $M$ - the number of discrete points in frequency range: $\omega=0 \div \omega_{M}$.

From here, we obtain square error between output response and real data:

$$
\sigma^{2}(C)=\int_{0}^{T}[y(t)-x(C, t)]^{2} d t \rightarrow C \min
$$

where $T$ - the amount of time to observ e the random output data series of real objects.

Regarding the discrete points of time series, we obtain minimization objective function:

$$
\sigma^{2}(C)=\sum_{i=1}^{N}\left[y\left(t_{i}\right)-x\left(C, t_{i}\right)\right]^{2} \rightarrow \min _{\mathbb{C}}
$$

where $N$ - the number of discrete points in an interval of observ ed time: $t=0 \div T$.

Objective function's value $\sigma^{2}(\mathbb{C})$ is determined after a computing process in the following order:

$$
\begin{gathered}
\mathbb{C} \rightarrow W(\mathbb{C}, \varrho+j \infty) \rightarrow X(\mathbb{C}, \varrho+j \infty) \rightarrow \\
P(\mathbb{C}, \omega) \rightarrow x(\mathbb{C}, t) \rightarrow \sigma^{2}(\mathbb{C})
\end{gathered}
$$

Therefore, $\sigma^{2}(\mathbb{C})$ is a computable function and is continuous and differentiable everywhere. On the other hand, is obvious non-linear functions to parameters and especially have the complex cleft(ravine) characteristic. With these characteristics, the most effective methods to solve the minimized problem (8) is to apply "cleft-over" optimization algorithm $[7,8]$.

The solution to the (8) problem with the selected structure $(m, n, q)$ of model (1) give us an optimal estimation $x\left(\mathbb{C}^{*}, t\right)$ with $y\left(t_{i}\right)$ series, and together with the optimal transfer function $W\left(\mathbb{C}^{*}, s\right)$ respectively.

\section{Determine the Optimal Estimation Model}

As above, with each selected struture $(m, n, q)$, an optimal solution is output response model $x\left(\mathbb{C}^{*}, t\right)$ and the $W\left(\mathbb{C}^{*}, s\right)$ optimal transfer function, respectively. Depending on the selected $(m, n, q)$ combination. There are infinite structures of the model. So, the facing problem is to find a $(m, n, q)$ struture so that the corresponding solution to the problem (8) brings out the response which is the proper estimation for the $y\left(t_{i}\right)$ time series.

According to $[1,2]$, the model is cosidered as a proper estimation if the obtained error series between the given model and time series become a radom distribution range in the form of "white noise". Assume a a significance level, the model is considered to be accurate if correlation coefficients value $r_{i}$ of error series satisfy the followed condition:

$$
\left|r_{i}\right| \leq u_{\frac{\alpha}{2}} / \sqrt{N}
$$

where $u_{\frac{\alpha}{2}}$ - is the limited value obeying the normal distribution rules, $N$ - the number of data sets of series.

On the other hand, the (1) model is fractional, so if we increase the $(m, n)$ degrees its accuracy will increase as a result. Particularly, $q$ is the nonstatic degree of model, it depends on the behaviour of output response and is equal to the degree of the 
asymptote of output response. $q=0$ if the asymptote of output response is horizontal asymptote. $q=1$ if the asymptote of output response is oblique asymptote. $q>1$ if output has no asymptote. In fact, $q \leq 1$ in most cases.

To define the global optimal estimation model, the steps to solve the identification problem are as follows:

(1) Select the degree of $q$ and fix it from the output response's behaviour.

(2) Exploratively select values of denominator's degree $n$, and values of nominator's degree $m=n-1$ simultaneously.

(3) For each selected $(m, n, q)$ structure we solve the $(8)$ estimation model problem.

(4) With the respective $W\left(\mathbb{C}^{*}, s\right)$ and $x\left(\mathbb{C}^{*}, t\right)$ obtained, we determine error series and check the condition upon model suitability.

(5) If the condition is satisfied, the obtained model is optimal and the respective $W\left(\mathbb{C}^{*}, s\right)$ transfer function is the solution to the identification problem.

(6) If the model is not suitable, we will select other models with $m$ and $n$ 's degree gradually increased and repeat from step 3 .

\section{Example}

Suppose the output signal of an implement controlled object is the step pulse in form (2). At that time, from the output, the measured response signal in form of time series is as follows:

The Graf of the time series $y(t)$ obtained from experiment is shown in Fig. 2.

\begin{tabular}{lllllllllll}
\hline$i$ & 1 & 2 & 3 & 4 & 5 & 6 & 7 & 8 & 9 & 10 \\
\hline$t_{i}$ & 0.0100 & 0.2054 & 0.4008 & 0.5962 & 0.7916 & 0.9870 & 1.1824 & 1.3778 & 1.5732 & 1.7686 \\
$y_{i}$ & -0.0023 & 0.0060 & 0.2540 & 0.6000 & 1.0641 & 1.3125 & 1.4700 & 1.4126 & 1.2500 & 1.2191 \\
\hline$i$ & 11 & 12 & 13 & 14 & 15 & 16 & 17 & 18 & 19 & 20 \\
\hline$t_{i}$ & 1.9640 & 2.1594 & 2.3548 & 2.5502 & 2.7457 & 2.9411 & 3.1365 & 3.3319 & 3.5273 & 3.7227 \\
$y_{i}$ & 1.0800 & 1.0830 & 1.0127 & 1.0300 & 0.9900 & 1.0310 & 0.9407 & 0.9900 & 0.9600 & 0.9740 \\
\hline$i$ & 21 & 22 & 23 & 24 & 25 & 26 & 27 & 28 & 29 & 30 \\
\hline$t_{i}$ & 3.9181 & 4.1135 & 4.3089 & 4.5043 & 4.6997 & 4.8951 & 5.0905 & 5.2859 & 5.4813 & 5.6757 \\
$y_{i}$ & 0.9500 & 0.9900 & 0.9560 & 0.9960 & 0.9539 & 1.0400 & 0.9680 & 1.0510 & 0.9900 & 1.0211 \\
\hline
\end{tabular}

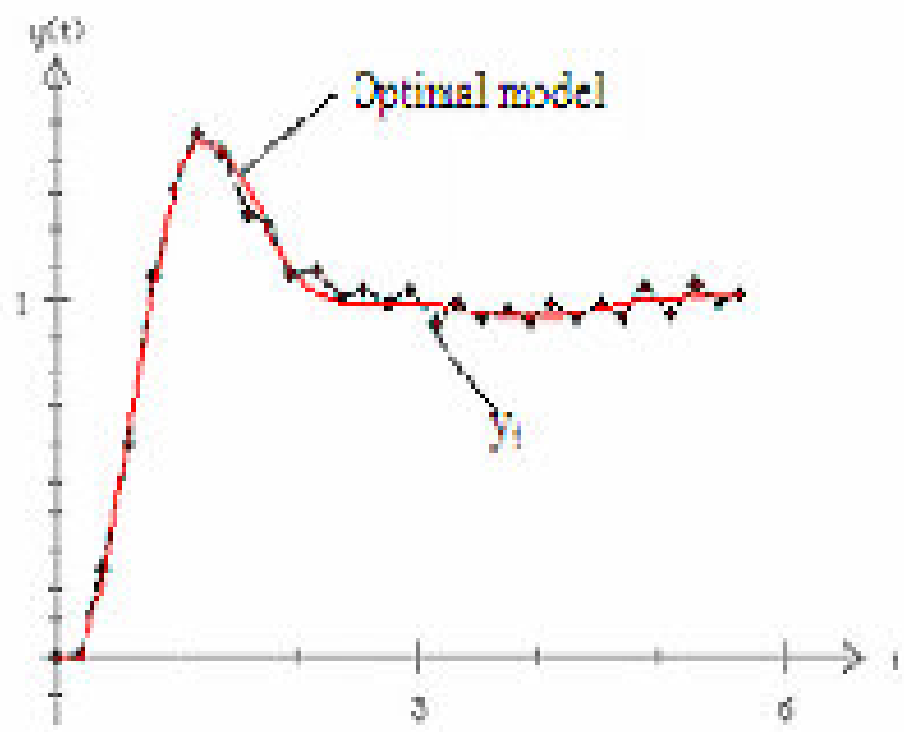

Fig. 2 The curve of output response data series of directive object. 
We identify the transfer function of object based on model (5) by solving the (8) problem with diffrent structures. The image of input signal step pulse: $U(s)=1 / s$. The behaviour of the data series above is corresponding to the nonstatic degree where $q=0$. Hence, we only have to select the suitable degree of numerator and denominator $(m, n)$ of tranfer function (5). Having selected the $(m, n)$ structure, we solve the (8) problem by the "cleft-over" algorithm $[7,8]$.

\subsection{Structures of Tested Models}

(1) The first structure, we choose: $m=0, n=1$. The respective optimal model is:

$$
W_{1}\left(\mathbb{C}^{*}, s\right)=\frac{b_{0}}{1+a_{1} s} \cdot e^{-\tau s}=\frac{1.065}{1+0.167 s} \cdot e^{-0.364 s}
$$

The error series between the output response $x_{1}\left(\mathbb{C}^{*}, t\right)$ and the measured data is on figure $3-\mathrm{a}$. The root-mean-square Error of the model is $\bar{\sigma}=0.1429$. By using this model, the estimation is obviously incorrect because the condition $\left|r_{i}\right| u_{\frac{\alpha}{2}} / \sqrt{N}$ is clearly not satisfied.

(2) The second structure, we choose: $m=0, n=2$. The optimal model is:

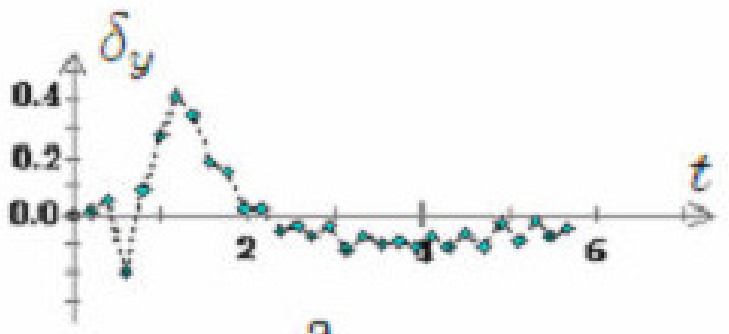

a.

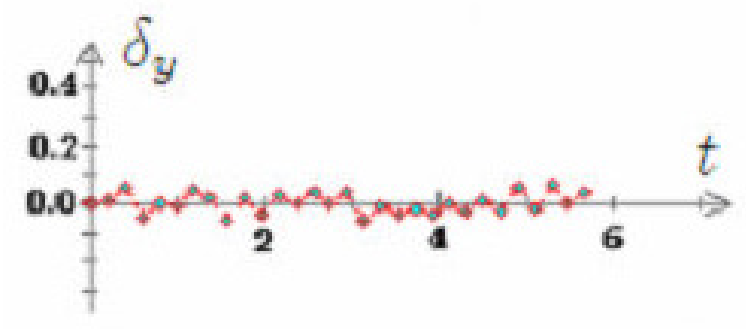

c.

$$
\begin{aligned}
& W_{2}\left(\mathbb{C}^{*}, s\right)=\frac{b_{0}}{1+a_{1} s+a_{2} s^{2}} \cdot e^{-\tau s}= \\
& \frac{1.033}{1+0.239 s+0.123 s^{2}} \cdot e^{-0.108 s}
\end{aligned}
$$

The error series between the output response $x_{2}\left(\mathbb{C}^{*}, t\right)$ and the measured data is on figure 3-b. The root-mean-square Error of the model is $\bar{\sigma}=0.0682$. This model brings out the incorrect estimation because the condition $\left|r_{i}\right|$ teqslantu $u_{\frac{\alpha}{2}} / \sqrt{N}$ is still not satisfied.

(3) With the third structure, we choose: $m=1, n=3$. The optimal model is:

$$
\begin{aligned}
& W_{3}\left(\mathbb{C}^{*}, s\right)=\frac{b_{0}\left(1+b_{1} s\right)}{\left(1+a_{1} s+a_{2} s^{2}\right)\left(1+a_{3} s\right)} \cdot e^{-\tau s} \\
& =\frac{0.985(1+1.166 s)}{\left(1+0.365 s+0.098 s^{2}\right)(1+0.683 s)} \cdot e^{-0.226 s}
\end{aligned}
$$

The error series between the output response $x_{3}\left(\mathbb{C}^{*}, t\right)$ and the measured data is on figure 3 -c. The root-mean-square Error of the model is $\bar{\sigma}=0.0342$. This model brings out the nearly correct estimation, the condition upon modelsuitability $\left|r_{i}\right| u_{\frac{\alpha}{2}} / \sqrt{N}$ is nearly satisfied.
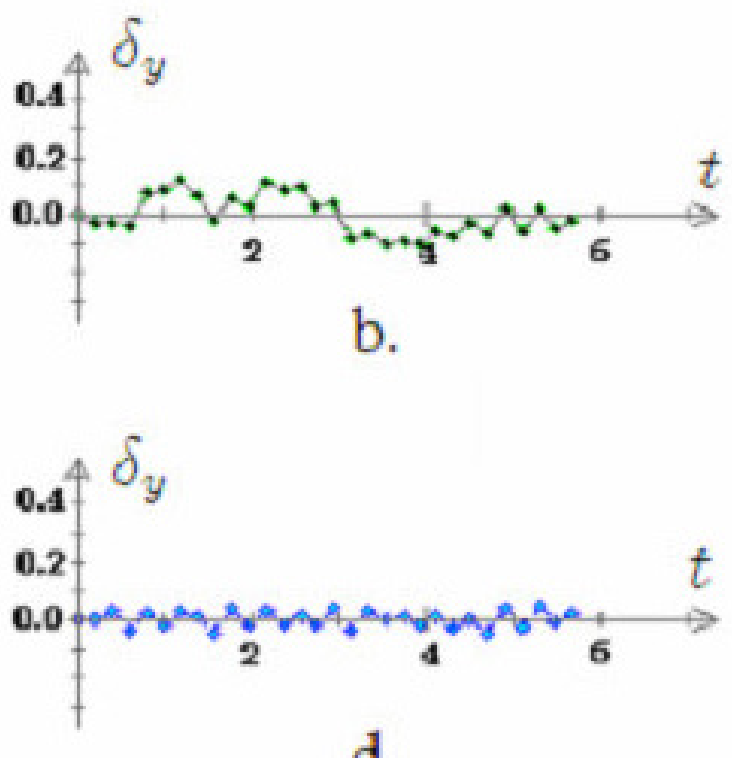

Fig. 3 The error series of models. 
(4) The fourth structure, we choose: $m=2, n=4$. The optimal model is:

$$
\begin{aligned}
& W_{4}\left(\mathbb{C}^{*}, s\right) \\
& =\frac{b_{0}\left(1+b_{1} s+b_{2} s^{2}\right)}{\left(1+a_{1} s+a_{2} s^{2}\right)\left(1+a_{3} s+a_{4} s^{2}\right)} \cdot e^{-\tau s} \\
& =\frac{1.001\left(1+0.716 s+0.37 s^{2}\right)}{\left(1+0.202 s+0.084 s^{2}\right)\left(1+0.545 s+0.414 s^{2}\right)} \cdot e^{-0.183 s}
\end{aligned}
$$

The error series between the output response $x_{4}\left(\mathbb{C}^{*}, t\right)$ and the measured data is on figure 3 -d. The root-mean-square Error of the model is $\bar{\sigma}=0.0212$. This model brings out the correct estimation since the condition upon model suitability $\left|r_{i}\right| u_{\frac{\alpha}{2}} / \sqrt{N}$ is completely satisfied.

While increasing the degrees of $m, n$ of the model, the error series is almost non-decreasing. In the optimizing process, the old coefficients are almost invariable, whereas the added coefficients - arising while increasing the degree of model - are always forced to zero by the algorithm. The optimal solution is nearly at a stand still. According to these results, the transfer function model $W\left(\mathbb{C}^{*}, s\right)$ has error series which is similar to "white noise", and simultaneously yields. The root-mean-square Error minimum. Therefore, we can considered it as the global optimal model of object. The respective output response of the model is shown on figure 2 .

\section{Conclusion}

(1) The objects with dead time delay are popular class of objects in industrial control, its transfer function has non-linear property for parameters, therefore classic identification methods have low effectiveness.

(2) Recommending using the transfer function with dead time delay as the basic model and by using the inverse Laplace transformation we obtain the output response of the model. On this basis, we solve the object identification problem in the form of the time series estimation problem based on measured random data of controlled object.

(3) The recommended method in this report enable us to solve the directive object identification problem generally and effectively under the random noise.

(4) The optimal model of object determined by the estimation method for non-linear random model ensures the suitability according to probability and statistic's point of view.

\section{References}

[1] G. Christian, A. Monfort, Times Series and Dynamic Models, Cambridge University Press, 1997.

[2] Nguyen Van Huu. Nguyen Huu Du, Statistic Analysis and Forecast, Hanoi National University Press, 2003. (in Vietnamese).

[3] P. EykhofF, System Identification, Wiley, 1974.

[4] P. EykhofF, Trends \& Progress in System Identification, Pergamon Press, 1981.

[5] A.A. Krasovski, Summarization of Development History and Situation of the Control Theory, Automation and Industry Jour., Moscow, No 6-7 (1999) 1. (in Russian).

[6] Nguyen Van Manh, A Frequency Method Calculating the Output Response of a Line Automatic Control System without Use of H-function Table, Informatic and Control Jour., Hanoi, No 1 (1995) 30. (in Vietnamese).

[7] Nguyen Van Manh, The Affine projection method for solving non-linear optimization problems. Proceeding of NCST of VietNam, No. 2 (1992) 53.

[8] Nguyen Van Manh, Methods Optimization of Control System for Uncertaint Processes, Dissertation of Science Doctor. Moscow Power Energy Institute, 1999. (in Russian). 